Frank Bash

Department of Astronomy, University of Texas

Austin, Texas

\title{
ABSTRACT
}

We describe a series of observational studies of M81 which are presently underway. We hope that these studies will allow improved understanding of this one, simple, density-wave spiral galaxy.

\section{RADIO MAPS}

Bash and Kaufman (Ohio State) have observed M81 using the VLA in the radio continuum at wavelengths of $6 \mathrm{~cm}$ and $20 \mathrm{~cm}$ using configurations $B, C$ and $D$, and in the polarization mode. At this time we have only preliminary results. The strongest source in M81 is the VLBI source at the center which has a flux density ranging from $\sim 50$ to $\sim 100 \mathrm{mJy} /$ beam-area depending on the frequency and configuration. Regions which are very bright on the Hodge and Kennicutt (1983) $\mathrm{H} \alpha$ plates and the central part show up also on the $20 \mathrm{~cm} \mathrm{B-configuration} \mathrm{maps} \mathrm{and} \mathrm{the} 6 \mathrm{~cm}$ D-configuration maps while essentially only the center can be seen at $6 \mathrm{~cm}$ on the B-configuration.

Perhaps the most interesting of our continuum maps up to this point is that made using the C-configuration at $20 \mathrm{~cm}$. Figure 1 shows that map. The faint radio emission along the arms between the HII regions can now be seen. Although it is saturated in Figure 1, the central part shows a source northeast of the center whose peak brightness is about $0.8 \mathrm{mJy} / \mathrm{beam}$ area. The VLA beam here is $12 ! .7 \times 12^{\prime !}: 5$ and the brightness of the central source is $\sim 90 \mathrm{mJy} /$ beam area.

In addition to the continuum study, Hine (Texas) and Rots (VLA) are making a HI, $21-\mathrm{cm}$ line map of M81 with the VLA's D and B configurations.

\section{HII-REGION RADIAL VELOCITIES}

Levreault, Leisawitz and Bash have observed 15 HII regions in M81 and measured their radial velocities to an accuracy of typically better than $10 \mathrm{~km} \mathrm{~s}^{-1}$. These data are partly reduced and they should allow us to check Bash and Visser (1981), who predict non-circular velocities of up to $20 \mathrm{~km} \mathrm{~s}^{-1}$. 
STELLAR-POPULATION SYNTHESIS

Hilton and Bash are studying the surface photometry, in the U, B, V and I bands, and spectrophotometry, with about $10 \mathrm{~A}$ resolution, of the spiral arms of M81 and NGC2841. This differs from the pioneering work of Schweizer (1976) in that it uses standard photometric bands, adds a longerwavelength filter and adds spectra. We desire to determine the stellar populations of the arms of M81 which are consistent with the observed photometry and spectroscopy, and to contrast those populations with the arms of NGC2841. NGC2841 is a classical chaotic, non-density-wave galaxy (Kormendy and Norman, 1979).

Much of this work has been supported by NSF grant AST-8116403.

\section{REFERENCES}

Bash, F. N., and Visser, H. C. D. 1981, Astrophys. J., 247, 488. Hodge, P. W., and Kennicutt, R. C., Jr. 1983, Astrophys. J., 267, 563. Kormendy, J. and Norman, C. 1979, Astrophys. J., 233, 539.

Schweizer, F. 1976, Astrophys. J. Supp1. 31, 313.

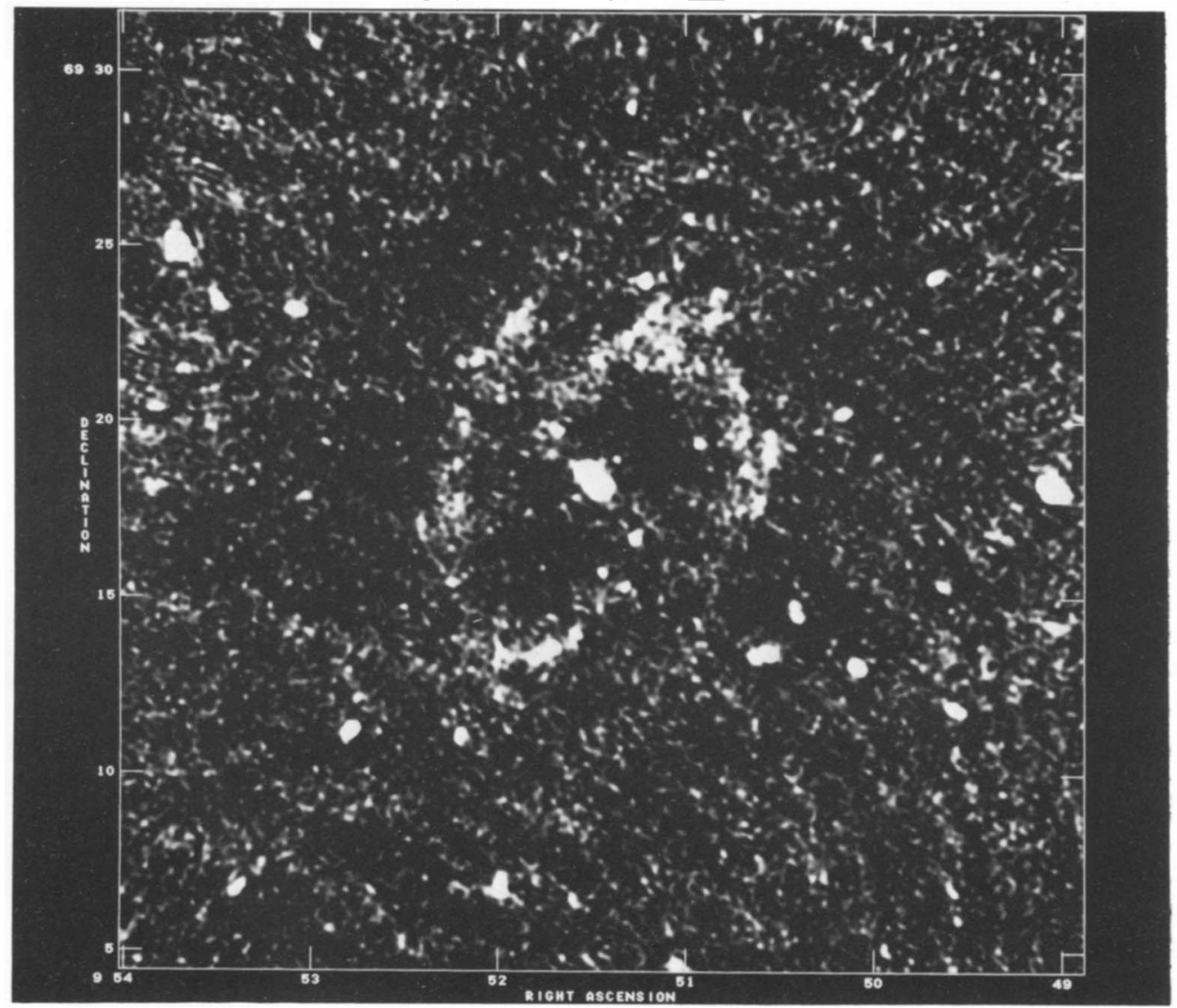

Fig. 1 - VLA radlo map of $\mathrm{M} 81$ at $20 \mathrm{~cm}$ using the C-configuration. The intensity scale saturates at $1 \mathrm{mJy} /$ beam-area; the noise is $60 \mu \mathrm{Jy} /$ beam-area. 